Article

\title{
Integration study, analysis and design of a flexible antenna in OLED light for WiMAX Wireless Communication Systems ${ }^{\dagger}$
}

\author{
Soukaina Sekkal ${ }^{1}$, Laurent Canale ${ }^{2,}$, Mariam El Gharbi ${ }^{3}$ and Adel Asselman ${ }^{1}$ \\ 1 Optics and Photonics Laboratory, Université Abdelmalek Essaadi Tetouan, Morroco; \\ sekkalsoukaina92@gmail.com, adelasselman@gmail.com \\ 2 LAPLACE, Université de Toulouse, CNRS, INPT, UPS, Toulouse France; \\ laurent.canale@laplace.univ-tlse.fr \\ 3 Information Technology \& Systems Modeling Team, Université Abdelmalek Essaadi Tetouan, Morroco; \\ elgharbimariam93@gmail.com \\ * Correspondence: laurent.canale@laplace.univ-tlse.fr; \\ + This paper is an extended version of our paper published in 2020 IEEE 20th International Conference on \\ Environmental and Electrical Engineering (EEEIC), Madrid, Spain, June 2020.
}

\begin{abstract}
In this work, a new flexible antenna integrated with OLED light sources is presented for WiMAX wireless communication systems. The proposed antenna was placed on a $100 \%$ polyester base with a thickness of $1.5 \mathrm{~mm}$ and achieved a high gain. We evaluated and tested its performance, including reflection coefficient, radiation pattern and gain. The flexible and simple patch antenna has been designed to operate at $3.5 \mathrm{GHz}$ for WiMAX wireless communication systems with a gain value of $5.38 \mathrm{~dB}$. This article proves the applicability of the proposed material for the integration of flexible antennas in OLEDs while maintaining gain performance similar to conventional flat antennas.
\end{abstract}

Keywords: flexible antenna; wireless communication systems; OLED; transparent conductive fabric; VeilShield

\section{Introduction}

In recent years, significant research efforts have been made to develop the integration of antennas in existing products because of the growing need for data throughput which will require the multiplication and diversification of communication channels. On the other hand, OLED technologies are capable of being the next generation of light sources with wide, diffuse and glarefree lighting [1], due to their efficiency, their long lifespan and their high quality of light and their easy-to- manufacture products. Although they are useful for lighting as well as for screens, extremely thin, potentially curved, flexible and even rollable. With the development of smart windows, we can for example mention windows will integrate OLED, as well as other versatile functions [2], [3]. In the same framework of integration, we present here the integration of a textile and flexible antenna in OLED light sources.

Future wireless communication systems employ several applications before dealing with applications which require high gain and system capacity. Textile antennas respond to the need to improve both system capacity and to have higher gain. Their property of flexibility made them initially favorable to communicate freely via a wireless network or by integrating them into clothing [4], [5].

Flexible antennas are made from different materials and vary in cost and performance which frequently must have additional features such as sturdiness and flexibility. Previous work has shown that a flexible antenna must be made of unconventional materials to meet current requirements which offer new trends in wireless communications such as conductive ink [6], silver paste [7], conductive 
fabrics [8] and conductive wires [9] used to design the antenna radiating element and polyester [10], the polymer, using cotton and felt [8], polydimethylsiloxane (PDMS) film and graphene film (FGF) [11] as substrate.

In this paper we present a simulation study of a flexible antenna in transparent conductive fabric VeilShield integrated in an OLED light source (Organic Light Emitting Diodes). The simple patch antenna has been proposed for high gain operation of $5.38 \mathrm{~dB}$ at $3.5 \mathrm{GHz}$ to be used for WiMAX Wireless communication systems. The present work is based on the process of adding flexibles antennas in OLED without affecting their performance. This work is organized as follows: in first part of Section II a simulation study of the flexible antenna is presented, In the second part, we integrated this antenna into the OLED light source structure.

\section{Materials and Methods}

In this section, we describe the flexible antenna design for WiMAX Wireless communication systems. One fully fabric antenna was designed to operate at $3.5 \mathrm{GHz}$ measured at $(|S 11|<-10 \mathrm{~dB})$ to demonstrate the concept of using this type of fabric to design flexible antennas. The antenna had a size of $55 \times \mathbf{4 6} \mathbf{m m}^{\mathbf{2}}\left(\boldsymbol{L}_{\boldsymbol{s}} \times \boldsymbol{W}_{\boldsymbol{S}}\right)$. The dimensions microstrip feed line are calculated to ensure a $50 \Omega$ input impedance with length and width value $\left(\boldsymbol{L}_{f}=\mathbf{1 4} \mathbf{~ m m}, \mathbf{W}_{\mathrm{f}}=\mathbf{4} . \mathbf{1 2} \mathrm{mm}\right)$ respectively. In the antenna geometry, the ground plane covers the full rear part of the substrate to reduce back radiation. For simulation, design and optimization CST (Computer Simulation Technology) Microwave Studio was used. The structure of proposed antenna and its parameters are shown in Figure 1, and Table 1, respectively.

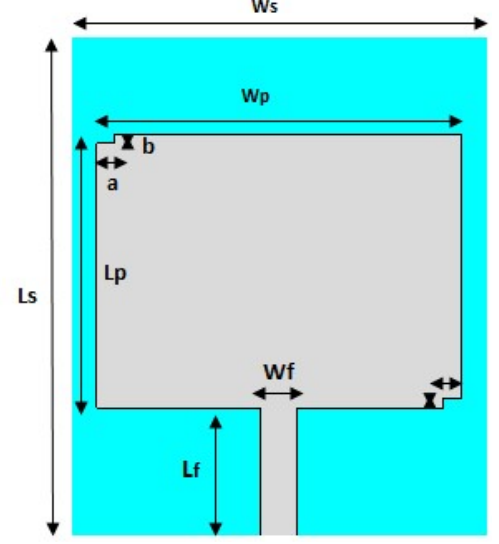

(a)

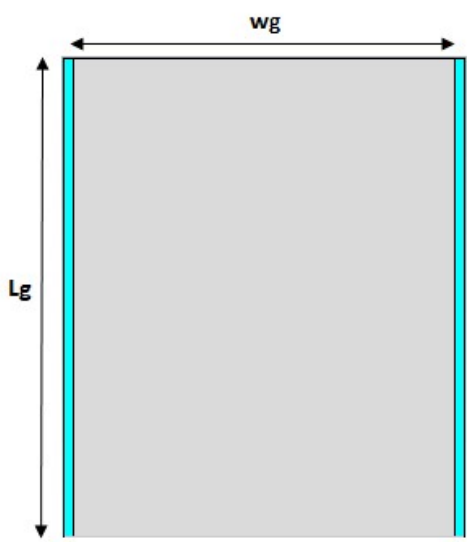

(b)

Figure 1. The geometry of the flexible antenna: (a) radiating patch; (b) ground plane.

Table 1. Parameters of the proposed antenna.

\begin{tabular}{cccc}
\hline $\begin{array}{c}\text { Design } \\
\text { parameters }\end{array}$ & Value(mm) & $\begin{array}{c}\text { Design } \\
\text { parameters }\end{array}$ & Value(mm) \\
\hline Wp & 40 & $\mathrm{Wg}$ & 40 \\
$\mathrm{Lp}$ & 30 & $\mathrm{Lg}$ & 55 \\
$\mathrm{a}$ & 2 & $\mathrm{~b}$ & 1 \\
\hline
\end{tabular}

For the conducting parts of the antenna (the radiating element and the ground plane) we have used the parameters of the transparent conducting fabric VeilShield. This fabric is made of woven mesh polyester fibers, coated with blackened nickel / zinc copper for greater corrosion resistance. it has a very low sheet resistance of $0.089 \Omega / s q$ with a thickness value of $0.057 \mathrm{~mm}$ and an electrical conductivity of $2.10^{5} \mathrm{~S} / \mathrm{m}$.

The calculated theoretical transparency of the fabric using [12] is $73 \%$.

$$
T \%=(p-s)^{2} / p^{2},
$$


Where s was the strip width had a size of $90 \mu \mathrm{m}$ and $\mathrm{p}$ is the pitch of the mesh with a size of $620 \mu m$, as shown in Figure 2.

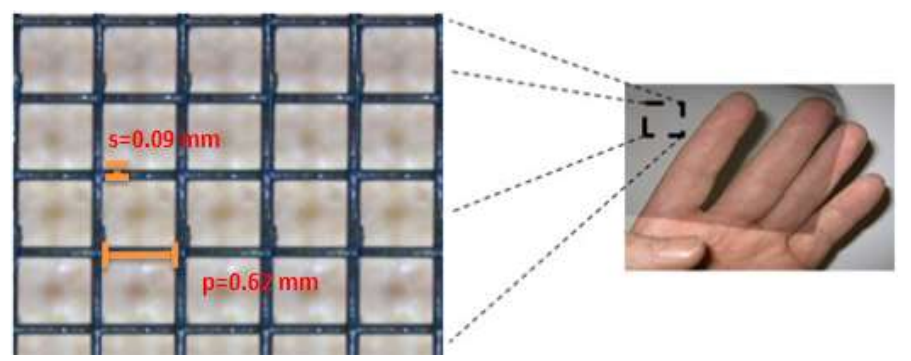

Figure 2. Transparent fabric tissue [13].

For the insulating element of the antenna we have used $100 \%$ Polyester. This fabric is made of polyester threads or fibers. Its dielectric constant is $\varepsilon=2.85$, with a loss tangent of 0.02 . In addition, polyester is flexible, very stable to moisture, having even greater resistance and elasticity to deformation of $40 \%$ [14].

\section{Results and discussions}

In this section we present the simulation results of the proposed antenna including reflection coefficient, gain, distribution of surface current and radiation pattern. In addition, we evaluated the functioning of the proposed structure by a parametric study.

\subsection{Parametric study}

\subsubsection{The variation of parameter $b$ :}

We also studied the variation of the length $b$, to see its effect on the parameter S11. Figure 3, shows the results of the reflection coefficient S11 with the variation of this parameter $(b=1 \mathrm{~mm}$, $\mathrm{b}=3 \mathrm{~mm}, \mathrm{~b}=5 \mathrm{~mm}$ ). From this figure, we observe that the resonant frequency of the antenna does not affect much on the variation of the parameter $b$, therefore we notice that when we increase the value of the parameter $b$ the resonance frequency increases a little bit as well as parameter S11 decreases by a small step.

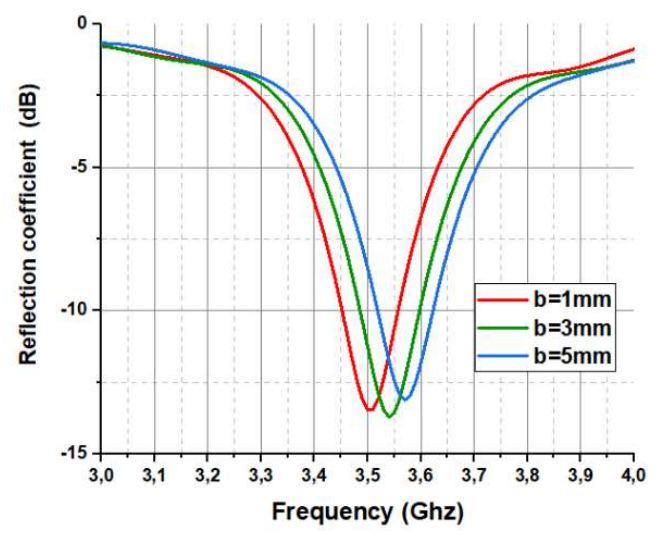

Figure 3. Reflection coefficient (S11) with the variation of the parameter b. 


\subsection{Reflection coefficient (S11) of the proposed antenna}

The performance of the proposed antenna in terms of reflection coefficient is demonstrated in Figure 4.

From the result of reflection coefficient of the proposed antenna can be note that the antenna is exhibiting resonance frequency $(\mathrm{S} 11 \leq-10 \mathrm{~dB})$ at $3.5 \mathrm{GHz}$ is obtained to operate for WiMAX Wireless communication systems.

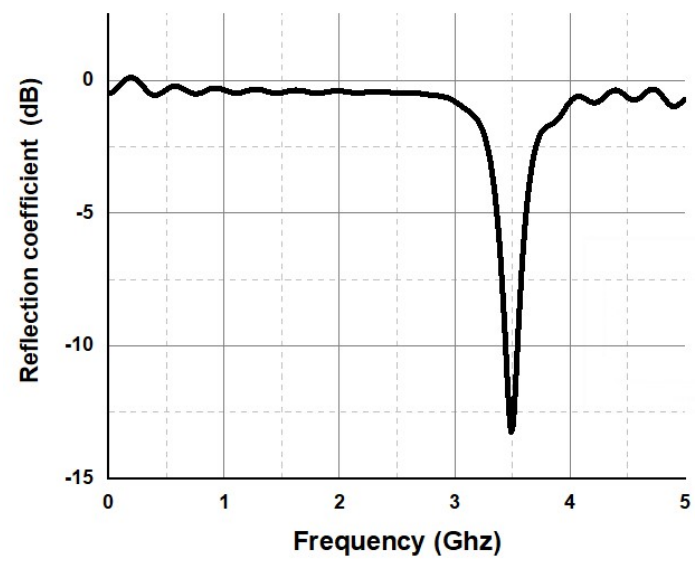

Figure 4. Performance of the proposed flexible antenna in terms of reflection coefficient.

\subsection{Gain of proposed antenna}

To know the amount of energy emitted by our antenna we focused the study on the gain of this proposed antenna. figure 5, shows the variation of the simulated gain of the flexible antenna with a frequency band from $3 \mathrm{GHz}$ to $3.8 \mathrm{GHz}$.

We can be can notice that the flexible antenna has a high gain of $5.38 \mathrm{~dB}$ at $3.5 \mathrm{GHz}$. The gain increases as the frequency increases.on the other hand in a certain moment the gain tends to decrease when the frequency exceeds the value $3.4 \mathrm{Ghz}$. It can be noted that the overall performance of the proposed design in terms of gain at the frequency $3.5 \mathrm{GHz}$ is better than in other bandwidths.

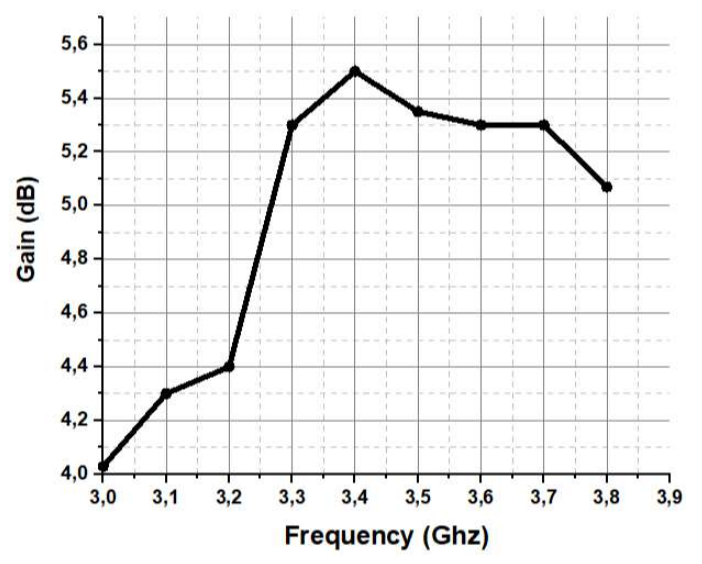

Figure 5. Performance of the proposed flexible antenna in terms of gain.

\section{4. $3 D$ radiation pattern of the proposed antenna}

We also studied the far field radiation pattern of the proposed antenna to evaluate its performance. The 3D radiation pattern of the flexible antenna at $3.5 \mathrm{GHz}$ is illustrated in Figure 6. 
In general, Figure 6 shows a stable power distribution at the resonant frequency of $3.5 \mathrm{GHz}$. As well as the results obtained have demonstrated that the flexible proposed antenna has forwardoriented radiation with a gain of $5.38 \mathrm{~dB}$ at $3.5 \mathrm{GHz}$.

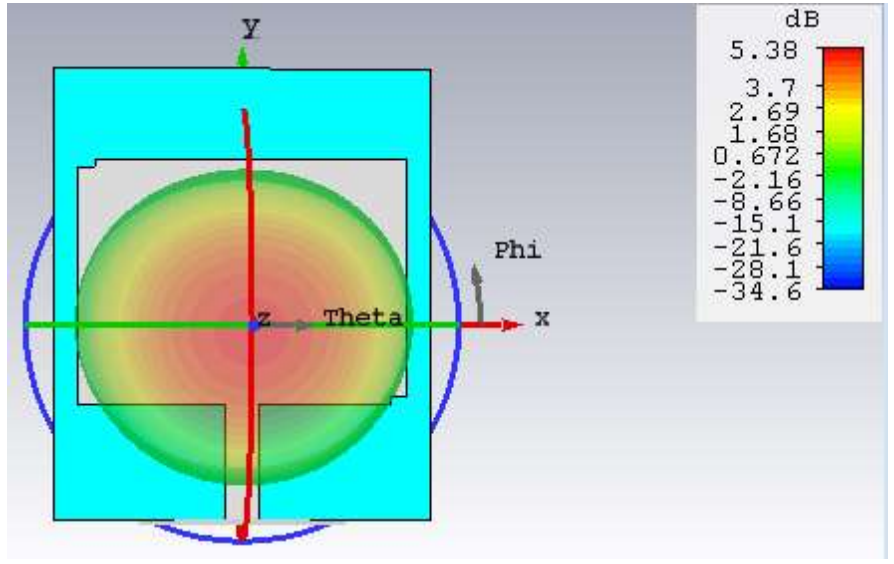

Figure 6. The 3D radiation pattern of the flexible antenna at $3.5 \mathrm{GHz}$.

\subsection{Integration of the flexible antenna into the structure of OLED}

This paper is the first to report the integration of a flexible antenna in OLED light sources. Our goal was to operate the proposed antenna in the OLED structure. The proposed principle allows OLEDs to function both as a light source and as a wireless data transmitter for WiMAX wireless communication systems.

\subsubsection{Geometry of the proposed antenna integration in OLED structure}

The proposed flexible antenna is integrated into an OLED of simple structure which contains 4 layers. Figure 8 , shows the structure of the OLED light source with the integrated antenna. The proposed antenna is placed in front face of the OLED.

In this work we have used the parameters of the OLED presented in [15], where the dimensions are $130.2 \times 47.8 \mathrm{~mm}^{2}$. The OLED layers are shown as follows: Layer 1 describes the aluminum cathode with a thickness of $120 \mathrm{~nm}$. Layer 2 describes the polymer with a thickness of $270 \mathrm{~nm}$. Layer 3 represents the anode of indium tin oxide (ITO) with a thickness of $150 \mathrm{~nm}$. Layer 4 has the glass substrate with a thickness of $215 \mathrm{~nm}$.

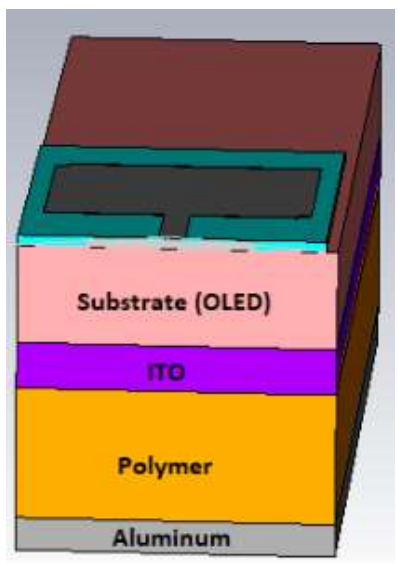

Figure 7. The OLED light source structure with the flexible antenna. 


\subsubsection{Reflection coefficient (S11) of the proposed antenna integration in OLED structure}

The performance of the proposed antenna in terms of the reflection coefficient (S11) with and without OLED is shown in figure 8. This figure presents a comparison between the reflection coefficients of the flexible antenna before and after its integration into OLED.

This result shows that the reflection coefficient S11 of the proposed antenna is affected after their integration into the OLED. This results in a small variation in the resonant frequency, notably increasing the resonant frequency by a small step. This makes the input impedance of the proposed antenna less suitable for $50 \Omega$.

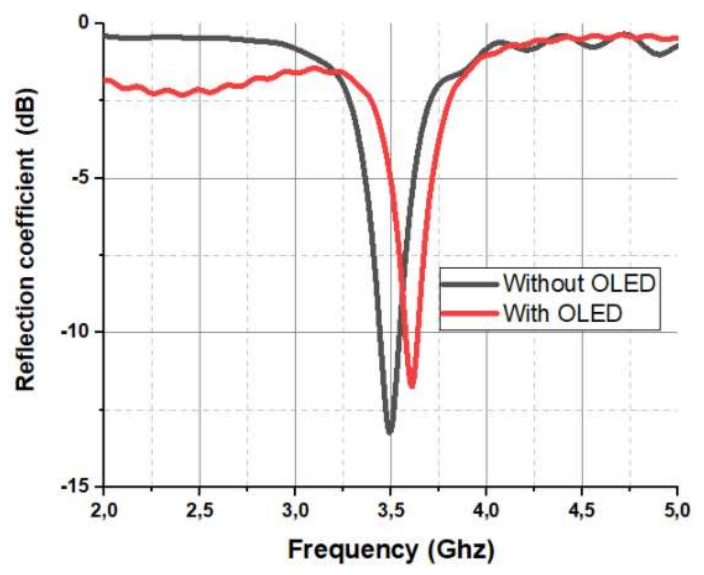

Figure 8. The reflection coefficient (S11) of the flexible antenna with and without OLED.

\subsubsection{Radiation pattern of the proposed antenna integration in OLED structure}

The operation of the proposed antenna integrated in the OLED with and without OLED in terms of radiation simulated at the resonant frequency $3.5 \mathrm{GHz}$ is illustrated in figure 9. This figure presents the comparison between the radiation patterns in polar coordinates of the proposed antenna with and without OLED. The results of the two planes $\mathrm{E}$ and $\mathrm{H}$ shows that the two radiation patterns have the same radiation direction and they are oriented upwards along the $\mathrm{Oz}$ axis.

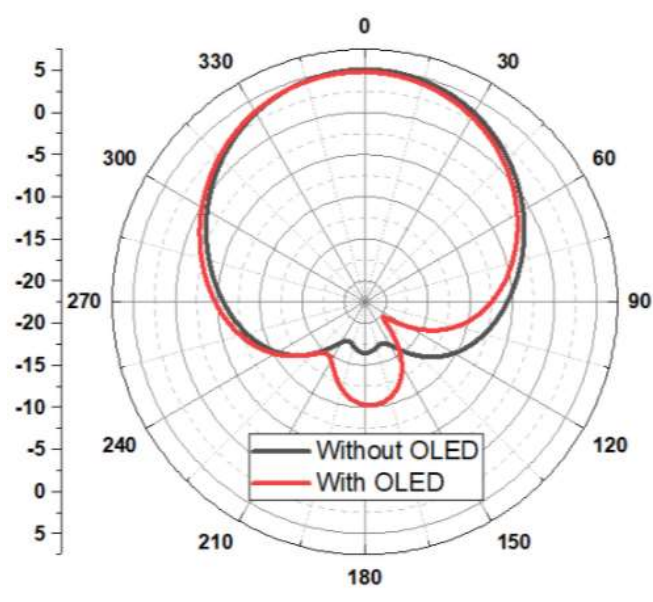

(a)

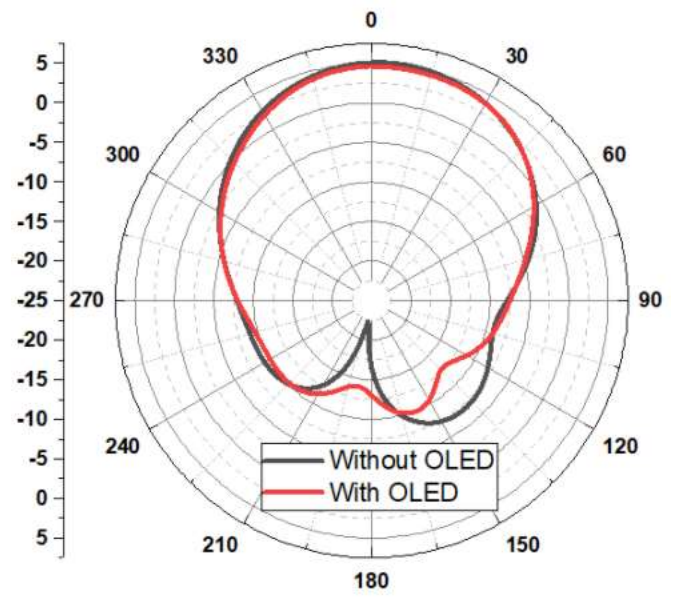

(b)

Figure 9. Radiation pattern of the proposed antenna with and without OLED at 3.5 GHz (a); E-Plane (b); H-

Plane. 


\section{Conclusions}

In this article, a new purely textile and flexible patch antenna has been designed and integrated into the structure of an OLED light source to work in WiMAX wireless communication systems at the resonant frequency 3.5 GHz. We have proposed to embedded in the same device both lighting and WiMAX wireless communication systems. The proposed antenna consists of different parts, the radiating element and the non-radiating element. The radiating element contains a rectangular patch with two slits on its ends. The non-radiating element contains a layer of the $100 \%$ polyester substrate which is placed between the radiating element and the ground plane. Our designed antenna is flexible and has simple structure and low weight.

We have shown that antenna design techniques work well even for flexible antennas using unconventional materials. The overall performance was analyzed in terms of the reflection coefficient, the gain and the radiation pattern.

Our proposed antenna works at $3.5 \mathrm{GHz}$ frequency with high gain of $5.38 \mathrm{~dB}$ even after integrating the antenna into the OLED device, these parameters are less affected by this device.

Author Contributions: Conceptualization, S.S. and M.E.G.; methodology, S.S. and M.E.G.; software, S.S. and M.E.G.; validation, S.S., M.E.G.; data curation, S.S. and M.E.G.; writing-original draft preparation, S.S. and M.E.G.; writing-review and editing, S.S., M.E.G., L.C. and A.A.; visualization, S.S., M.E.G., L.C. and A.A.; supervision, L.C., A.A.; project administration, L.C. and A.A. All authors have read and agreed to the published version of the manuscript.

Funding: This work was funded by French Ministry of Europe and Foreign Affairs and Moroccan Ministry of Higher Education and Scientific Research as a part of Program Hubert Curien "Maghreb" MELINA (Mastering Efficient Lighting in North Africa) granted to L.C.

Conflicts of Interest: The authors declare no conflict of interest.

\section{References}

[1] S.-J. Zou, Y. Shen, F.-M. Xie, J.-D. Chen, Y.-Q. Li, and J.-X. Tang, "Recent advances in organic light-emitting diodes: toward smart lighting and displays," Mater. Chem. Front., vol. 4, no. 3, pp. 788-820, 2020.

[2] B. D'Andrade and S. Forest, "Novel Smart Windows Based on Transparent Phosphorescent OLEDs,” Universal Display Corporation, 2006.

[3] L. Canale, "Imaginons l'éclairage du Futur ," Dossier Mieux Voir Et Bien-Etre, Magazine LUX. Magazine LUX, 2019.

[4] I. Locher, M. Klemm, T. Kirstein, and G. Troster, "Design and characterization of purely textile patch antennas," IEEE Trans. Adv. Packag., vol. 29, no. 4, pp. 777-788, 2006.

[5] Y. Ouyang, E. Karayianni, and W. J. Chappell, "Effect of fabric patterns on electrotextile patch antennas," in 2005 IEEE Antennas and Propagation Society International Symposium, 2005, vol. 2, pp. 246-249.

[6] L. Yang, A. Rida, R. Vyas, and M. M. Tentzeris, "RFID tag and RF structures on a paper substrate using inkjet-printing technology," IEEE Trans. Microw. Theory Tech., vol. 55, no. 12, pp. 2894-2901, 2007.

[7] S.-J. Ha and C. W. Jung, "Reconfigurable beam steering using a microstrip patch antenna with a U-slot for wearable fabric applications," IEEE Antennas Wirel. Propag. Lett., vol. 10, pp. 1228-1231, 2011.

[8] R. Cicchetti, E. Miozzi, and O. Testa, "Wideband and UWB antennas for wireless applications: A comprehensive review," Int. J. Antennas Propag., vol. 2017, 2017.

[9] S. Zhang et al., "Embroidered wearable antennas using conductive threads with different stitch spacings," in 2012 Loughborough Antennas \& Propagation Conference (LAPC), 2012, pp. 1-4.

[10] S. Sankaralingam, S. Dhar, and B. Gupta, "Preliminary studies on performance of a $2.45 \mathrm{GHz}$ wearable antenna in the vicinity of human body," in 2012 International Conference on Communications, Devices and Intelligent Systems (CODIS), 2012, pp. $250-253$.

[11] M. El Gharbi, R. Fernández-García, S. Ahyoud, and I. Gil, “A Review of Flexible Wearable Antenna Sensors: Design, Fabrication Methods, and Applications,” Materials (Basel)., vol. 13, no. 17, p. 3781, 2020.

[12] J. Hautcoeur et al., "Transparency and electrical properties of meshed metal films," Thin Solid Films, vol. 519, no. 11, pp. 3851$3858,2011$.

[13] S. Sekkal, L. Canale, and A. Asselman, "Flexible Textile Antenna Design with Transparent Conductive Fabric Integrated in OLED for WiMAX Wireless Communication Systems," in 2020 IEEE International Conference on Environment and Electrical 
Engineering and 2020 IEEE Industrial and Commercial Power Systems Europe (EEEIC/I\&CPS Europe), 2020, pp. 1-4.

[14] S. Martin Rey and F. Romana Melodia, "Mechanical strength parameters on polyester fabrics. Treatment applied in support of large canvas painting:" the expulsion of the merchants from the temple" by artist Saverio Lillo.(1734-1796)," Arché, no. 4-5, pp. 139-146, 2010.

[15] A. Alchaddoud, L. Canale, G. Ibrahem, and G. Zissis, "Photometric and Electrical Characterizations of Large-Area OLEDs Aged Under Thermal and Electrical Stresses," IEEE Trans. Ind. Appl., vol. 55, no. 1, pp. 991-995, 2018. 